\title{
Electroinduced drift of solvated ions in salt solution of Ce and Ni
}

\author{
HONGDA LI $^{\mathrm{a}}$, MISHIK KAZARYAN ${ }^{\mathrm{b}}$, IGOR SHAMANIN ${ }^{\mathrm{c}}$, SERGEY TIMCHENKO ${ }^{\mathrm{c}, *(\mathbb{D}) \text { and }}$ \\ IVAN USHAKOV \\ ${ }^{a}$ Department Equipment Engineering, Shenyang Ligong University, Shenyang 110159, China \\ ${ }^{b}$ Optics Department, P. N. Lebedev Physical Institute of the Russian Academy of Sciences, 53 Leninskiy \\ Prospekt, Moscow, Russia 119991 \\ ${ }^{\mathrm{c}}$ Laboratory for Isotopic Analysis and Technologies, Tomsk Polytechnic University, 30 Lenina Avenue, Tomsk, \\ Russia 634050 \\ E-mail: timsn@tpu.ru
}

MS received 26 March 2018; revised 9 April 2018; accepted 11 April 2018; published online 2 August 2018

\begin{abstract}
This paper shows the selective drift of cationic aqua complexes of chloride salts of metal ions in aqueous solutions under the exposure an asymmetric electric field $(100 \mathrm{~Hz}, 750 / 150 \mathrm{~V})$. The spatial distribution of $\mathrm{Ce}^{3+}$ and $\mathrm{Ni}^{2+}$ cations was demonstrated, and the maximum cation separation coefficients in the longitudinal section of the inter-electrode space were calculated for the stationary and circulating solution for different exposure durations of the electric field and solution circulation rates. A decrease in the relative concentration of cations was observed when moving from top down of the experimental cell, while the direction of cation drift along the sectional plane was independent of the distance from electrodes, the solution circulation rate, and nature of metal. It was established that while the solution moved from the grounded electrode to the potential electrode along the bottom and top parts of the sections, there was a decrease of concentrations of both cations in the central part of the cell and an increase near the electrodes. On the basis of the obtained data, the optimal points of sampling and drop were determined. The calculated maximum separation coefficients of $\mathrm{Ce}^{3+}$ and $\mathrm{Ni}^{2+}$ ions were $1.200 \pm 0.108$ and $1.036 \pm 0.025$, for the stationary and circulating solution at a rate of $7 \mathrm{~L} \cdot \mathrm{h}^{-1}$, respectively.
\end{abstract}

Keywords. Ion drift; salt solution; solvated ion; external electric field; asymmetric electric field.

\section{Introduction}

The phenomenon of selective drift of solvated ions (SDSI) in polar dielectric solvents has a number of promising technological applications. Thus, SDSI can be used to separate liquid radioactive wastes ${ }^{1}$ and rare earth elements ${ }^{2}$ in combination with other methods. ${ }^{3-9}$ Despite the prospects, the efficiency of SDSI-based technologies requires the provision of conditions for the maximum demonstration of separation effects caused by the phenomenon. Wherein, the efficiency is understood as the amount of the released component of the mixture per unit time, which in turn depends on the intensity, the frequency of the electric field, the geometry of the separating elements, and the pumping rate of the mixture to be separated, which are required to optimize the SDSI phenomenon.

\footnotetext{
*For correspondence
}

Today, there is no clear understanding of the processes occurring in solutions of polar dielectrics under the influence of an asymmetric electric field. Earlier it was theoretically shown ${ }^{10,11}$ that the total energy of a solvated ion-cluster, characterized by the state of the system, consists of the following: the energy of translatory motion of the entire cluster; the electron energy, determined by the quantum state of the electron shells of the ion and the solvent molecules associated around the ion; the vibrational energy, caused by the oscillation of the ion and the solvate shell relative to each other; ${ }^{4}$ and the rotational energy, corresponding to the rotation of the entire cluster or its parts relative to each other. ${ }^{5}$ Wherein, the inertial properties of clusters can differ for reasons of the difference in the ion masses while the coordination numbers are equal. For example, for a mixture of lithium isotopes aqua complexes $\left(\left[\mathrm{Li}^{2}\left(\mathrm{OH}_{2}\right)_{4}\right]^{+}\right.$and $\left.\left[\mathrm{Li}^{7}\left(\mathrm{OH}_{2}\right)_{4}\right]^{+}\right)$, SDSI can be used for enrichment of the required isotope. If there is a mixture of calcium and magnesium cationic aqua complexes 
$\left(\left[\mathrm{Ca}^{40}\left(\mathrm{OH}_{2}\right)_{4}\right]^{2+}\right.$ and $\left.\left[\mathrm{Mg}^{24}\left(\mathrm{OH}_{2}\right)_{4}\right]^{2+}\right)$ - SDSI is applicable for the separation of calcium and magnesium cations. In the case with anions, SDSI is also effective: for example, the $\mathrm{SO}_{4}^{2-}$ ion forms isotopically different aqua complexes $\left[\mathrm{S}^{32} \mathrm{O}_{4}\left(\mathrm{OH}_{2}\right)_{6}\right]^{2-}$ and $\left[\mathrm{S}^{34} \mathrm{O}_{4}\left(\mathrm{OH}_{2}\right)_{6}\right]^{2-}$ and the dissolution of the nitrate and periodate mixture results in the formation of a mixture of aqua complexes $\left[\mathrm{NO}_{3}\left(\mathrm{OH}_{2}\right)_{4}\right]^{-}$and $\left[\mathrm{IO}_{4}\left(\mathrm{OH}_{2}\right)_{4}\right]^{-}$. In the case with sulfur, SDCI can be used to separate the $S^{32}$ and $S^{34}$ isotopes, and in the case of nitrogen-iodine, for selective purification of the solution from nitrate or periodate. The third reason for the difference in the inertial properties of clusters is the difference in the coordination numbers of the ions which can be used for elemental enrichment in the solution.

The inertial properties of clusters determine the parameters of their motion trajectories in the solution under the action of an external periodic electric field. Wherein, the qualitative separation/purification requires data on the spatial distribution of solvated ions which occurs under the action of an asymmetric electric field. The trajectory of the aqua complex movement is determined by many parameters: the intensity and frequency of the field; the asymmetry coefficient and the bond length in the dipole (polarized aqua-complex); the mass ratio of the positive and negative parts of the dipole; the mass of aqua-complex as a whole; the polarization coefficient of the aqua complex, and others. However, there is still insufficient empirical data in the literature that would allow predicting the spatial distribution of metal cations in a common solution under different experimental conditions.

Therefore, the goal of this work is the experimental determination of the spatial distribution of metal cations under the action of the asymmetric electric field in a stationary and circulating solution to select the drop point of the mixture with the maximum cation separation coefficient.

\section{Experimental}

To determine the spatial distribution of solvated cations with different inertial properties in the volume of a solution placed between two parallel flat electrodes, there was developed a cell shown in Figure 1. The cell consisted of identical sections (height $-135 \mathrm{~mm}$, length $-150 \mathrm{~mm}$, width $-35 \mathrm{~mm}$ ) placed between potential electrodes. The section walls of $6 \mathrm{~mm}$ thick were made of polymethylmethacrylate. A copper foil (thickness $-0.6 \mathrm{~mm}$ ) was used as electrodes, it was located outside the sections and further isolated by polymethylmethacrylate (thickness $-6 \mathrm{~mm}$ ). The sections were separated by impermeable baffles, and their number was selected to accommodate the data reproducibility.

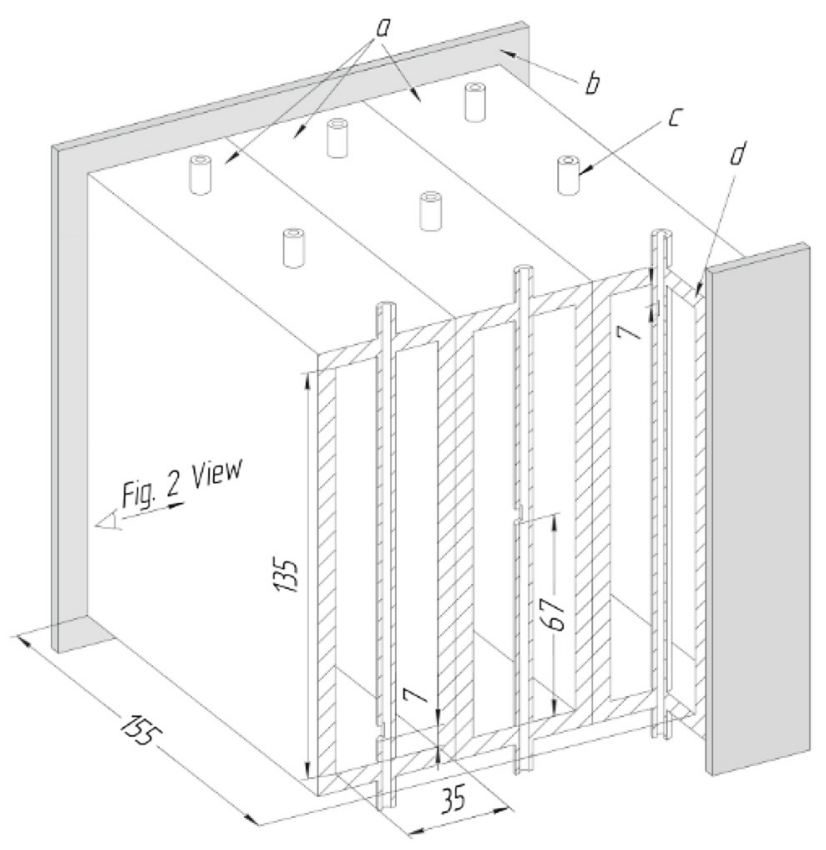

Figure 1. Experimental cell: $\mathrm{a}$ - sections; $\mathrm{b}$ - flat copper electrode; $\mathrm{c}$ - branch pipes for solution sampling; $\mathrm{d}$ - electrode isolation (polymethylmethacrylate).

The cation distribution was analyzed in the plane of the longitudinal profile of each cell part (Figure 2). In order to minimize the disturbance while perturbation was introduced into cation distribution during sampling, the solution was taken at three points in each of the three isolated sections operating under identical conditions. When comparing all the results, it could be seen that the sampling was made from the points at the top (Figure 2, points 1, 2 and 3), central (Figure 2, points 4, 5 and 6) and lower (Figure 2, points 7, 8 and 9) parts of the section. To provide data reproducibility all cells were duplicated.

Solution sampling was carried out automatically through a flow meter washer of rigidly fixed plastic capillaries (branch pipes) located in three different positions in each section.

We used the mixture of $\mathrm{Ce}$ and $\mathrm{Ni}$ ions for the research since indeed its separation from nitric acid solution is needed in spent fuel in reactors, otherwise, the metal ions absorb neutrons. The initial solutions were prepared on the basis of distilled water and salts of $\mathrm{CeCl}_{3} \cdot 7 \mathrm{H}_{2} \mathrm{O}$ and $\mathrm{NiCl}_{2} \cdot 7 \mathrm{H}_{2} \mathrm{O}$ (purity 99.9\%). The concentration of metal ions in the prepared solutions was chosen based on the requirement of correspondence to an ideal solution ${ }^{7,12}$ and was $1 \mathrm{~g} / \mathrm{L}$ for $\mathrm{Ce}^{3+}$ and $5 \mathrm{~g} / \mathrm{L}$ for $\mathrm{Ni}^{2+}$. The frequency of the electric field was $100 \mathrm{~Hz}$; ambient temperature $-22 \pm 0.2{ }^{\circ} \mathrm{C}$; the amplitude of the field strength in the positive half-period $-56 \mathrm{~V} / \mathrm{cm}$; the ratio of positive half-cycle amplitude to the amplitude of the negative field was $750 / 150 \mathrm{~V}$. The duration of the electric field exposure in the experiments was 0.5, 1 and $2 \mathrm{~h}$. Each experiment was performed three times.

The experiment was performed in two modes: in a stationary solution and with the solution circulating through a section of the experimental cell. In the first case, the cell was filled 


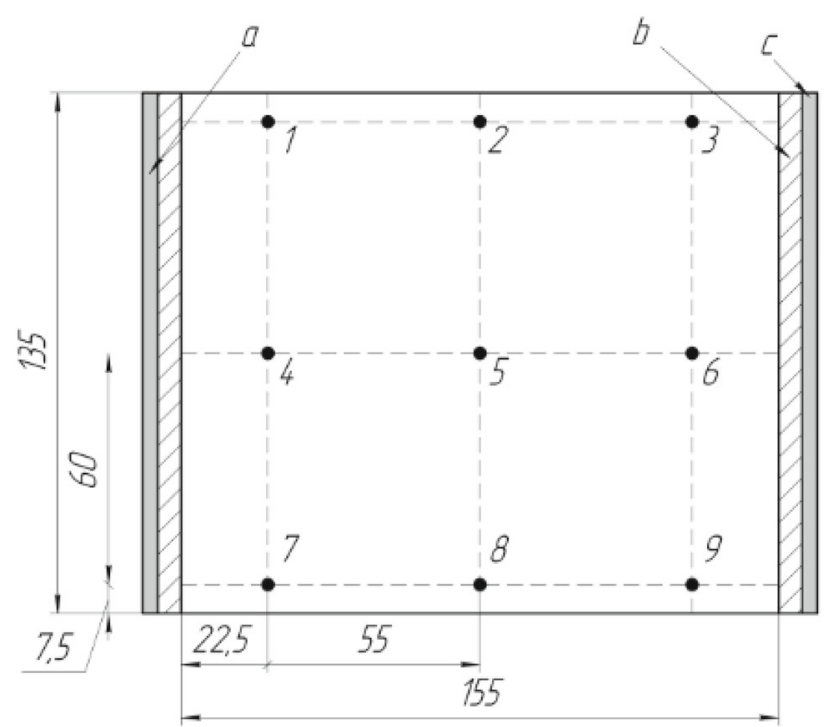

Figure 2. Longitudinal profile of experimental section: a - grounded electrode; b - electrode isolation (polymethylmethacrylate); $\mathrm{c}$ - potential electrode; 1,2,3...9 - sampling points.

with a solution prior to the experiment. In the second case the salt solution was fed via a peristaltic pump, with the solution circulation rate in the cell of $1.4,7$ and $14 \mathrm{~L} \cdot \mathrm{h}^{-1}$, into a point 1 (Figure 2), and outputs from the cell through the point 9. The solution sampling was made from point 7 , the drop was taken to the point 2 (Figure 2). When the solution circulation rate was over $7 \mathrm{~L} \cdot \mathrm{h}^{-1}$, the determined concentrations varied within the analysis error, so their analysis was not considered.

The metal concentration in the salt solution was determined using an iCAP6300Duo atomic emission spectrometer (ThermoFisher Scientific, USA) with inductively coupled plasma. The calibration curves were built with the help of multielement solutions. The range of determined concentration was $0.1 \ldots 20000 \mathrm{mg} / \mathrm{L}$ for both ions. Obtained spectra were processed by iTEVA Program Soft. The error in determining the metal concentration did not exceed $8 \%$.

The resulting concentration of metal in the solution was used to calculate the relative concentration of cations at a given point as a ratio $R_{i j}^{\prime}=C_{i}^{\prime} / C_{j}^{\prime}$, where $C$ is concentration and $\mathrm{i}$ and $\mathrm{j}$ are indices for cerium and nickel ions, respectively. The relative concentration of cations was used to calculate the maximum cation separation coefficient in the mixture of salts by formula $\alpha_{i j}=\frac{R_{i j}^{\prime}}{R_{i j}}$.

\section{Results and Discussion}

\subsection{Experiment with the stationary solution}

It was experimentally established that the relative content of cations in the analyzed section plane depends on the nature of the cations and the sampling point.

From the results it can be seen that when the salt solution is exposed by asymmetric electric field, the drift of cerium and nickel cations along the sectional plane has a similar character: a decrease in the relative concentrations of ions was observed down up due to the drift of cations to the bottom of the section. For example, the relative content of cations of a grounded electrode in the top down direction is $89,102,115 \%$ and $94,101,106 \%$ for $\mathrm{Ce}^{3+}$ (Figure 3a) and $\mathrm{Ni}^{2+}$ (Figure 3b), respectively. The distribution pattern along the section (top down) is similar for the central part and near the potential electrode.

As the solution moves from the grounded electrode to the potential electrode along the bottom and top parts of the sections, there is a decrease of the concentrations of both cations in the central part of the section and its increase near the electrodes. Wherein, a greater content of ions was accumulated near the potential electrode. Nevertheless, the character of the distribution in the transverse direction depends on the sampling height: in the equidistant from the electrodes the concentration of ions varies slightly and amounts $98 \ldots 103$ and $100 . .101 \%$ for $\mathrm{Ce}^{3+}$ and $\mathrm{Ni}^{2+}$, respectively.

An analysis of the influence of cation nature showed that there is a noticeable impoverishment of cerium cations (18.78\%) in the top part of the section (Figure $3 \mathrm{a}$, point 2 ), while nickel cations at a given point are slightly impoverished $(9.15 \%$ ) (Figure $3 b$, point 2$)$. An opposite tendency is noted in the bottom part of the section: enrichment with $\mathrm{Ce}^{3+}$ cations $7 \ldots 14 \%$ at points 7 and 9 is noted, while $\mathrm{Ni}^{2+}$ concentration increased by $2 . . .7 \%$ (Figure $3 b$, points 7 and 9 ).

Further, if we maintain the parameters of electric field and change the exposure duration for $30 \mathrm{~min}$ or $2 \mathrm{~h}$, separation of ions was not observed since the changes in concentration are within the error limits of the method.

\subsection{Experiment with the circulating solution}

The results of the experiments in the circulating solution mode are in good agreement with the results obtained in the experiments with the stationary solution. Thus, there is a slight increase in the concentration of $\mathrm{Ce}^{3+}$ and $\mathrm{Ni}^{2+}$ cations in the bottom section in the area of the ground electrode, up to $102 \ldots 103$ and $101 \%$, respectively (Figure 4, points 7 and 9). Conversely, there is a slight decrease of concentrations at the top of the: $\mathrm{Ce}^{3+}$ - up to $97 \%, \mathrm{Ni}^{2+}$ - up to $99 \%$ (Figure 4 , point 2 ). Thus, when the solution was circulating at a rate of $7 \mathrm{~L} \cdot \mathrm{h}^{-1}$, the main direction of the solvated cation drift was retained: towards the bottom of the cell.

The solution mobility did not affect the fact that the relative change in the $\mathrm{Ce}^{3+}$ concentration at the bottom of the cell exceeds the change in the $\mathrm{Ni}^{2+}$ concentration. For example, with a high circulation rate of the solution 


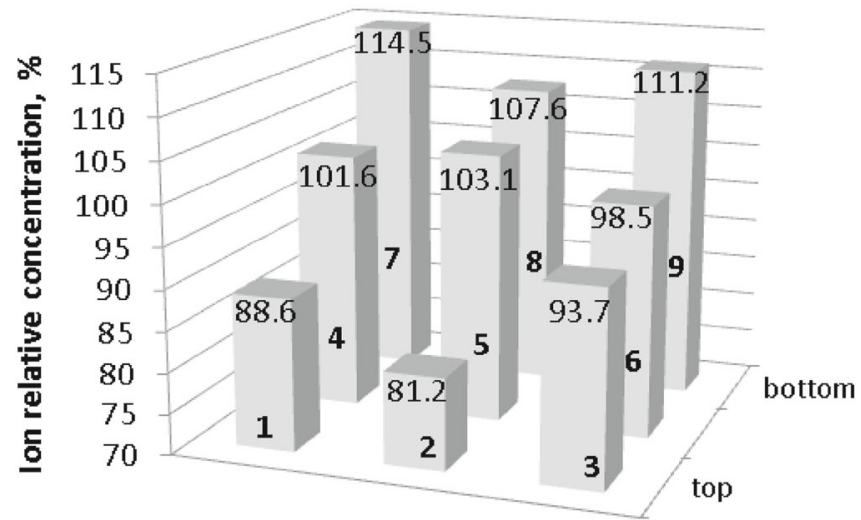

(a)

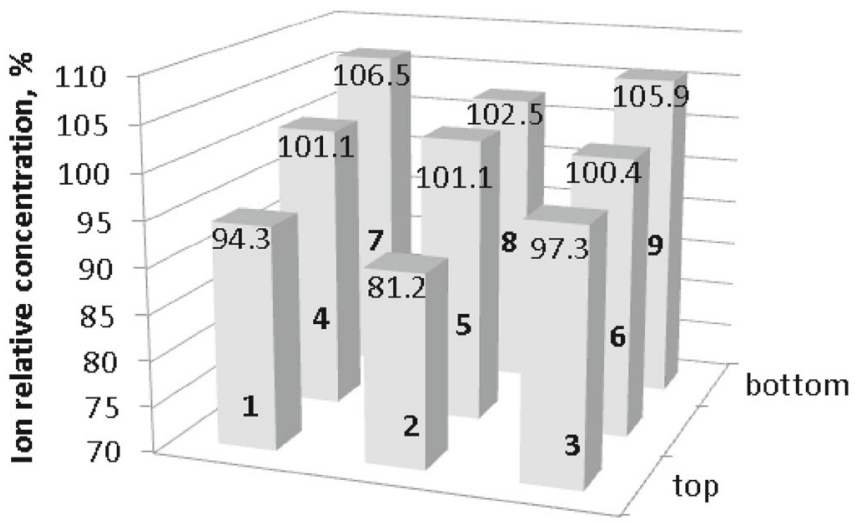

(b)

Figure 3. Distribution of $\mathrm{Ce}^{3+}(\mathrm{a})$ and $\mathrm{Ni}^{2+}$ (b) in sampling points $1 \ldots 9$ of a cell.

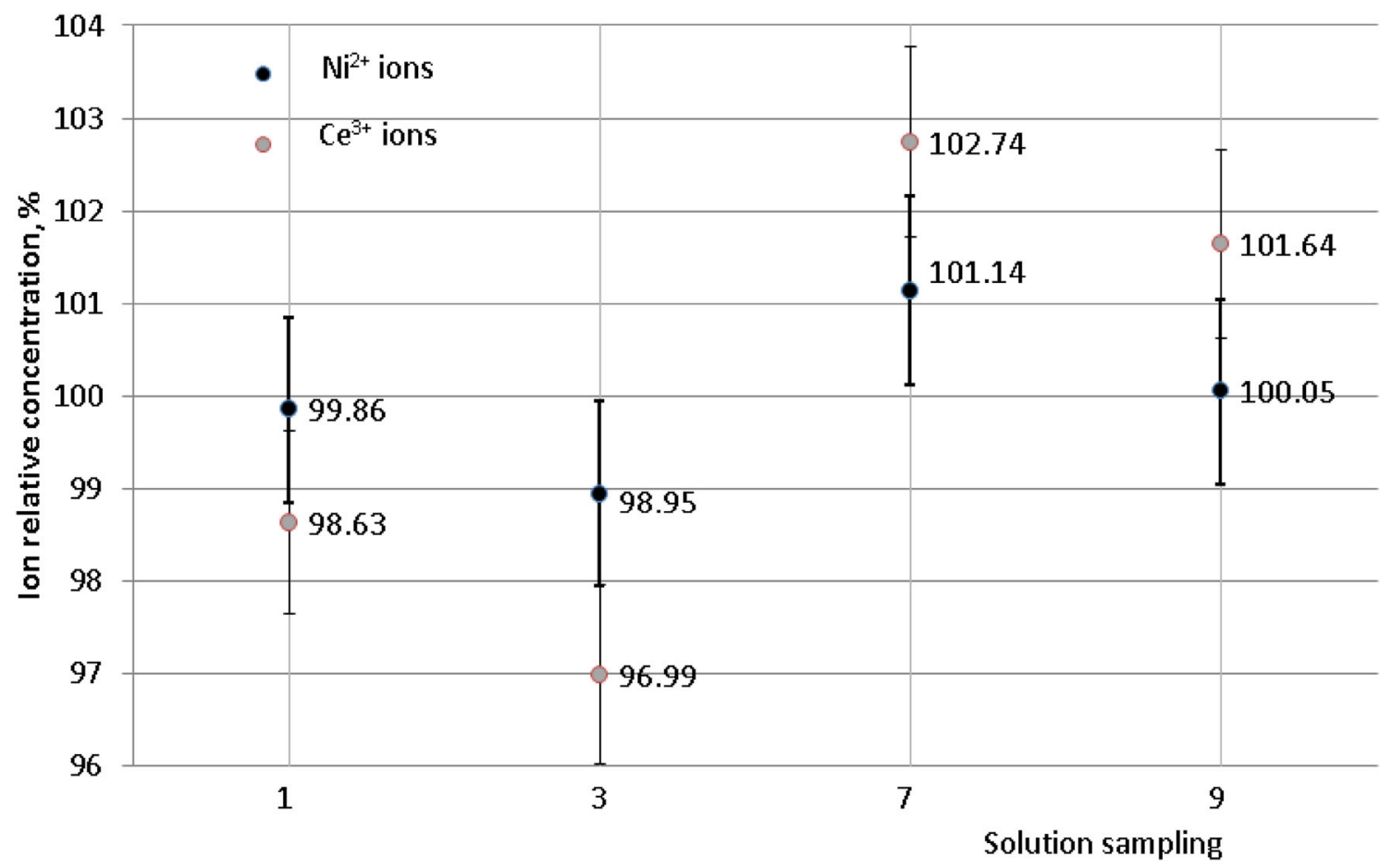

Figure 4. Ion relative concentration at solution circulation rate $7 \mathrm{~L} \cdot \mathrm{h}^{-1}$.

the increase of $\mathrm{Ce}^{3+}$ content is $2-3 \%$ compared to $\mathrm{Ni}^{2+}$ - less than $1 \%$ (Figure 4, points 7 and 9).

When reducing the solution circulation rate to $1.7 \mathrm{~L} \cdot \mathrm{h}^{-1}$, the general direction of the cationic aqua complexes, drift is selectively preserved and depends on the solution sample point. In general, it can be said that the relative concentration of cations at the sampling points is weakly dependent on the sampling point and varies between 98.6 to $101.8 \%$ and 99.2 to $100.7 \%$ for $\mathrm{Ce}^{3+}$ and $\mathrm{Ni}^{2+}$, respectively. Thus, at a low solution circulation rate, the intensity of the solvated cations distribution in salt solution falls significantly (Figure 5).

\subsection{Maximum cation separation coefficient}

The effect of the solution circulation mode was shown at the value of the maximum separation coefficient of the cation mixture. The optimal points of sampling, drop and feed were determined in stationary solution layer based on experimental results. The concentration components in the sampling are $C_{i}^{\prime}$ and $C_{j}^{\prime}$, and in the drop$C_{i}^{\prime \prime}$ and $C_{j}^{\prime \prime}$, for $\mathrm{Ce}^{3+} \mathrm{Ni}^{2+}$, respectively. The sampling is enriched with $\mathrm{Ce}^{3+}$ cations and impoverished with $\mathrm{Ni}^{2+}$ cations, and in the drop, on the contrary, it is impoverished with $\mathrm{Ce}^{3+}$ cations and enriched with $\mathrm{Ni}^{2+}$ cations. 


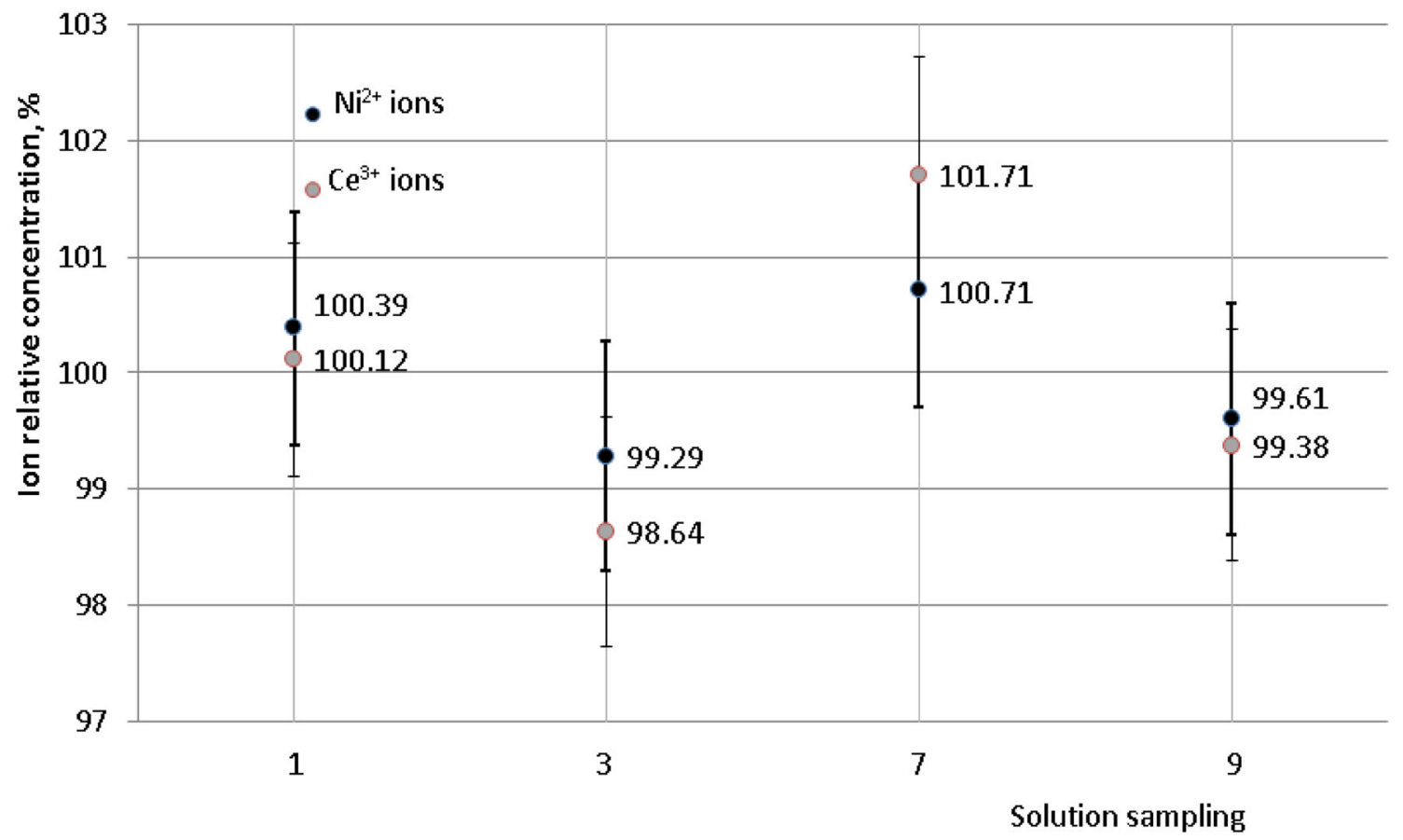

Figure 5. Ion relative concentration at solution circulation rate $1.7 \mathrm{~L} \cdot \mathrm{h}^{-1}$.

Table 1. The values of separation coefficients of $\mathrm{Ce}^{3+}$ and $\mathrm{Ni}^{2+}$ cations under the asymmetric field exposure $(100 \mathrm{~Hz}, 750 / 150 \mathrm{~V})$.

\begin{tabular}{llll}
\hline The solution circulation rate, $\mathrm{L} \cdot \mathrm{h}^{-1}$ & Drop at point 1 & Drop at point 2 & Drop at point 9 \\
\hline 14 & $1.021 \pm 0.036$ & $1.019 \pm 0.051$ & $1.034 \pm 0.051$ \\
7 & $1.029 \pm 0.019$ & $1.036 \pm 0.025$ & $1.000 \pm 0.017$ \\
1.7 & $1.014 \pm 0.021$ & $1.018 \pm 0.024$ & $1.014 \pm 0.019$ \\
0 & $0.894 \pm 0.011$ & $0.894 \pm 0.011$ & $0.894 \pm 0.011$
\end{tabular}

The maximum separation coefficient can be obtained if the sampling is taken at point 7 (Figure 2, bottom of the section at the potential electrode), and the drop - at point 2 (Figure 2, top part of the section). Thus, after an hour of exposure of field of 750/150 $\mathrm{V}$ with a frequency of $100 \mathrm{~Hz}$ to the aqueous solution of $\mathrm{CeCl}_{3}$ and $\mathrm{NiCl}_{2}$ salts mixture, the concentration values without circulation of the solution through the section are: $C_{i}^{\prime}=1.1454 ; C_{j}^{\prime}$ $=1.1065 ; C_{i}^{\prime \prime}=0.8122 ; C_{j}^{\prime \prime}=0.9085$. While the relative concentrations of $\mathrm{Ce}^{3+}$ and $\mathrm{Ni}^{2+}$ cations are: $R_{i j}=$ 1.00000 (initial solution); $R_{i j}^{\prime}=C_{i}^{\prime} / C_{j}^{\prime \prime}=1.0755$ (sampling) and $R_{i j}^{\prime \prime}=C_{i}^{\prime \prime} / C_{j}^{\prime \prime}=0.8940$ (drop). The obtained separation coefficients without solution circulating are shown as follows:

- in the sampling, $\alpha_{i j}=\frac{R_{i j}^{\prime}}{R_{i j}}=1.0755 \pm 0.097$,

- in the drop, $\beta_{i j}=\frac{R_{i j}}{R_{i j}^{\prime \prime}}=1.1186 \pm 0.1$,

- full coefficient, $q_{i j}=\alpha_{i j} \cdot \beta_{i j}=1.2027 \pm 0.108$.

The values of the separation coefficients were calculated based on the values obtained in the experiments.
Point 7 (Figure 2) was taken as 'sampling', in which the maximum difference in the concentrations of $\mathrm{Ce}^{3+}$ and $\mathrm{Ni}^{2+}$ cations is observed. The values of the separation coefficients for the selection of the "drop" solution at various points are given in Table 1 .

The values given in Table 1 suggest that the stable separation of $\mathrm{Ce}^{3+}$ and $\mathrm{Ni}^{2+}$ cations can be achieved at the circulation rate of $7 \mathrm{~L} \cdot \mathrm{h}^{-1}$, providing the drop from point 2 in the top part equidistant from the electrodes (Figure 2).

The maximum separation coefficient of solvated cations of cerium and nickel in the case of aqueous solution of their chlorides was achieved in experiments under the exposure of electric field of $750 / 150 \mathrm{~V}$ with a frequency of $100 \mathrm{~Hz}$ to the aqueous solution during an hour.

The obtained experimental results convincingly prove separation of solvated ions under the action of asymmetric low-frequency electrical field. The separation effect can be explained by the distance between homogeneously distributed ions, about $100 \mathrm{~nm}$, that corresponds 
to the coordination number of $10 .{ }^{13,14}$ This value is much higher of estimated radii for the first and the second solvated ion shell. ${ }^{14,15}$ Due to strong inverse relationship between field action radius and screening constant, we have the severe resonance between external field and ion radius fluctuations. Besides, in highly concentrated salt solutions, owing to the collision of ions, there are ion clusters having joint solvated shell.

\section{Conclusions}

It has been shown that under the exposure of asymmetric electric field $(100 \mathrm{~Hz}, 750 / 150 \mathrm{~V})$ to aqueous solutions of chloride salts of cerium and nickel, the selective drift of cationic aquacomplexes is induced. The spatial distribution of $\mathrm{Ce}^{3+}$ and $\mathrm{Ni}^{2+}$ cations has been experimentally demonstrated, and the maximum cation separation coefficients in the plane of the longitudinal section of the interelectrode space at the given conditions in the stationary and circulating solution have been calculated, for different durations of the electric field exposure and with different rates of the solution circulation.

According to the data of atomic emission spectroscopy, a decrease in the relative concentration of metal cations is observed when moving from top down of the developed experimental set up, while the direction of solvated cations drift along the sectional plane is independent of the distance from the electrodes, the solution circulation rate and the nature of the metal. It was established that while the solution moves from the grounded electrode to the potential electrode along the bottom and top parts of the sections, there is a decrease of the concentrations of both cations in the central part of the section and it is increased near the electrodes.

On the basis of the obtained data, the optimal points of sampling and drop are determined, for which the calculated maximum separation coefficients of $\mathrm{Ce}^{3+}$ and $\mathrm{Ni}^{2+}$ ions are $1.200 \pm 0.108$ and $1.036 \pm 0.025$, respectively, for the stationary and circulating solution at a rate of $7 \mathrm{~L} \cdot \mathrm{h}^{-1}$, respectively.

The performed experiments prove the possibility of using the phenomenon of electroinduced selective drift of solvated ions in salt solutions under the exposure of an asymmetric electric field for organizing the technological process of enrichment of target metal ions in solutions. However, successful development of such technology needs more experimental results on the separation of multicomponent systems.

\section{References}

1. Abdel Rahman $\mathrm{R} \mathrm{O}$, Ibrahium $\mathrm{H}$ A and Hung Y-T 2011 Liquid radioactive wastes treatment: a review Water 3551

2. Sun X, Do-Thanh C-L, Luo H and Dai S 2014 The optimization of an ionic liquid-based TALSPEAK-like process for rare earth ions separation Chem. Eng. J. 239392

3. Toktasynova A Z, Ahmetsharinova T E, Moreva I V, Yegorov I S and Sohoreva V V 2016 Isotopes: Technologies, materials and application September 19-23, 2016 (Tomsk Poytechnic Univ., Tomsk, Russia) p. 62

4. Ryabchikov A I, Golovkov V M, Stepanov I B, Sohoreva V V and Shulepov I A 2010 Modification of Materials with Particle Beams and Plasma Flows (Institute of Optical Atmosphere, Tomsk, Russia) p. 591

5. Karengin A G, Karengin A A, Podgornaya O D and Shlotgauer E E 2014 Complex utilization of SNF processing wastes in air plasma of high-frequency torch discharge IOP Conf. Ser.: Mater. Sci. Eng. 66012034

6. Karengin A G, Karengin A A, Novoselov I Y and Tundeshev N V 2015 Mater. Res. Adv. Mater. Res. 1084 158

7. Hofer T S, Tran H T, Schwenk C F and Rode B M 2004 Characterization of dynamics and reactivities of solvated ions by ab initio simulations J. Comput. Chem. 25211

8. Akimov D V, Djyachenko A N, Egorov N B and Zhuravlev N A 2016 Separation of rare earth elements by zone recrystallization IOP Conf. Ser.: Mater. Sci. Eng. 112012014

9. Egorov N B, Djyachenko A N, Akimov D V, Kiselyov A D, Obmuch K V and Chalov S A 2014 Extraction of REE by using solutions of $\mathrm{H}_{2} \mathrm{SO}_{4}$ and $\mathrm{NH}_{4} \mathrm{~F}$ Procedia Chem. 1115

10. Shamanin I V, Kazaryan M A, Timchenko S N and Poberezhnikov A D 2017 Equation of solvated ion oscillations in solution under an external periodic electric field B. Lebedev Phys. Inst. 44308

11. Shamanin I V, Kazaryan M A, Timchenko S N and Ushakov I A 2017 Mechanistic models of the ionsolvation shell system B. Lebedev Phys. Inst. 44254

12. Nightingale Jr E R 1959 Phenomenological theory of ion solvation effective radii of hydrated ions $J$. Phys. Chem. 631381

13. Kazaryan M A, Shamanin I V, Lomov I V, Dolgopolov S Yu and Lobanov A N 2011 Formation of associates of solvated cations in salt solutions in polar dielectric liquids B. Lebedev Phys. Inst. 38247

14. Gupta T and Rajaraman G 2014 How strongly are the magnetic anisotropy and coordination numbers correlated in lanthanide based molecular magnets? J. Chem. Sci. 1261569

15. Sarkar A, Chatterjee A, Tiwari S C and Tembe B L 2016 $\mathrm{Na}^{+} \mathrm{Cl}^{-}$ion pair association in water-DMSO mixtures: effect of ion pair model potentials J. Chem. Sci. 1281003 